\title{
Tunable quantum switcher and router of single atoms using localized artificial magnetic fields
}

\author{
Yan-Jun Zhao, ${ }^{1, *}$ Ning Tan, ${ }^{1}$ Dongyang Yu, ${ }^{2}$ Boyang Liu $\odot,{ }^{3}$ and Wu-Ming Liu ${ }^{2,4}$ \\ ${ }^{1}$ Faculty of Information Technology, College of Microelectronics, Beijing University of Technology, \\ Beijing 100124, People's Republic of China \\ ${ }_{2}^{2}$ Beijing National Laboratory for Condensed Matter Physics, Institute of Physics, Chinese Academy of Sciences, Beijing 100190, China \\ ${ }^{3}$ Institute of Theoretical Physics, Beijing University of Technology, Beijing 100124, China \\ ${ }^{4}$ School of Physical Sciences, University of Chinese Academy of Sciences, Beijing 100190, China
}

(Received 10 April 2020; accepted 19 August 2020; published 29 September 2020)

\begin{abstract}
We propose to generate localized artificial magnetic fields using two thin Raman laser beams intersected at a narrow region of a two-leg ladder, where the frequency difference must approximately match the energy offset between the two legs. Based on this method, we investigate the single-atom transport in a two-leg ladder with only two rungs, which, together with the legs, enclose a localized artificial magnetic flux. Here, the atoms on the two legs (channels) possess different onsite energies that produce another energy offset. We find that the atom incoming from the left channel can experience from blockade to transparency via modifying the onsite energy, tunneling strength, or magnetic flux, which can be potentially used for a quantum switcher. Furthermore, the atom incoming from the left channel can also be perfectly routed into the right leg, when, intriguingly, the outgoing atom in the right channel possesses a quasimomentum that can be modulated by the magnetic flux. The result may be potentially used for the interface that controls the communication between two individual quantum devices of cold atoms. The method can also be generalized to other artificial quantum systems, such as superconducting quantum circuit systems, optical systems, etc.
\end{abstract}

DOI: 10.1103/PhysRevResearch.2.033484

\section{INTRODUCTION}

The artificial tunability has made ultracold atoms an attractive platform for conducting research on quantum information processing [1-4] and high-precision instruments [5-9]. Integrating different cold atom systems together, one can possibly realize the scalable quantum network, with each quantum node being a cold atom subsystem, thus promoting the recent development of atomtronic devices [10-19]. However, reaching this destination demands the coherent manipulation of cold atom matter waves such that different nodes can be individually addressed for the effective communication of quantum signals. The atomtronic device that realizes such function is the atom switcher (router) for node-to-node(s) connection.

The exploration of atom switcher and router can benefit from the research on cold atom transport. It has been shown [20-35] that the transport properties can be tuned by atom interactions [36-39]. For example, spinless [20] and spinful [21] matter wave switchers have been proposed, besides which, there are also the studies on solitons in nonlinear lattices [26], transport in discrete breathers [32,34] and so on.

\footnotetext{
*Corresponding author: zhao_yanjun@bjut.edu.cn

Published by the American Physical Society under the terms of the Creative Commons Attribution 4.0 International license. Further distribution of this work must maintain attribution to the author(s) and the published article's title, journal citation, and DOI.
}

The researches on atomic switchers [20,21] mainly use the principle that a weak incident wave interacts with a strong Bose-Einstein condensate (BEC) that has to be deliberately prepared to tune the transport properties. This, however, has the drawback that the BEC can acquire accumulating momentum from the incident wave if it comes as successive pulses.

Moreover, to practically control the signal flow in quantum network, the indispensable quantum router needs to have multiaccess channels (rather than the one-channel atom switchers), which has been studied in the photonic system [40-45] but lacks investigation in cold atom systems.

Recently, we have noted that the artificial effective magnetic field, besides inducing topological physics [46-49], can also tune the transport properties of cold atoms, such as resulting in the novel chiral currents [50-53]. Inspired by this, we will explore a single-atom quantum switcher and router controlled by the localized artificial magnetic fields that coherently couple two individual atomic channels together, which model is equivalent to a two-leg ladder [50] with only two rungs. In detail, we first consider a ladder whose legs (channels) are decoupled due to an energy offset between them. To restore the localized interleg tunnelings, we propose to use two thin Raman laser beams intersected at a narrow region of the ladder, whose frequency difference must approximately match the energy offset between legs. When the interleg tunnelings are restored, an artificial magnetic flux, which originates from the spatial variation of the laser phases, will also be involved along a particular loop. Based on this method, we can create a two-leg ladder model where the artificial magnetic flux only penetrates one particular loop enclosed 


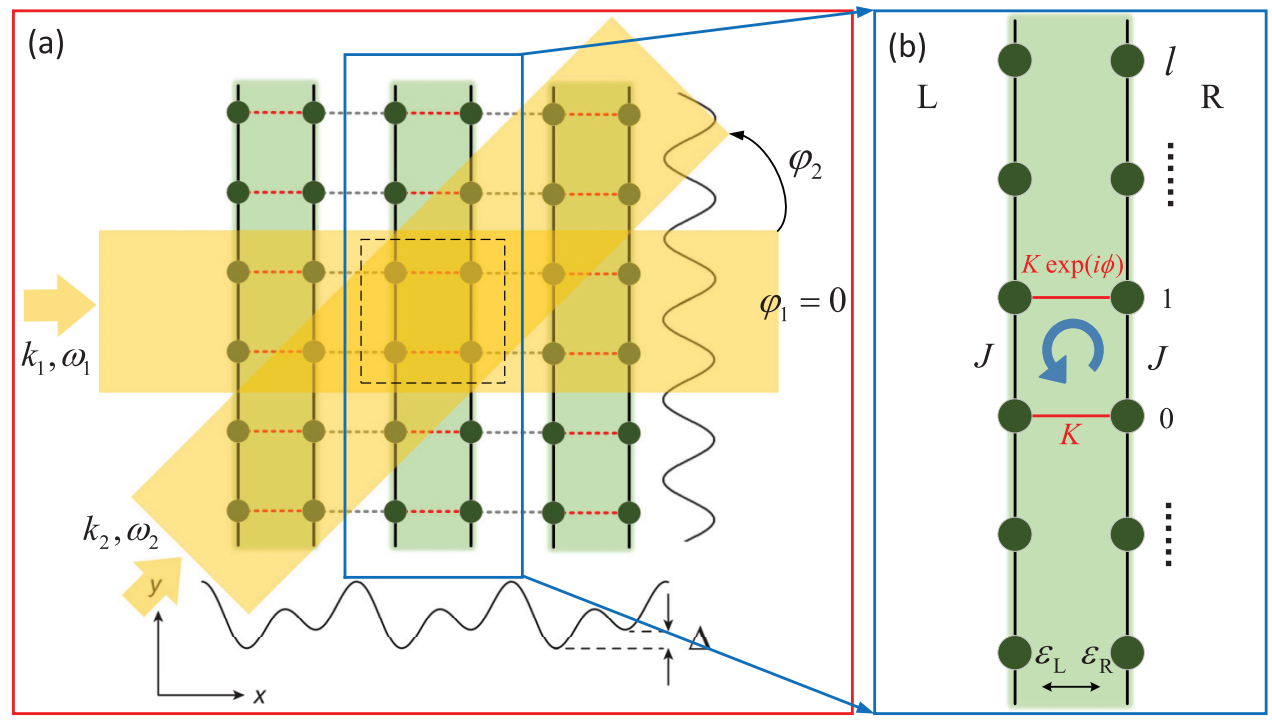

FIG. 1. (a) Experimental realization of localized effective magnetic fields using two thin Raman lasers and (b) the resulting two-leg ladder model with only two-rungs. The double-well potential generated in the horizontal direction produce an array of ladders, where the tunneling between adjacent legs is inhibited due to the energy offset $\Delta$. To restore the tunneling, we apply two lasers respectively incoming at the angles $\varphi_{1}$ (conveniently, $\varphi_{1}=0$ is specified here) and $\varphi_{2}$, whose frequency difference $\omega=\omega_{2}-\omega_{1}$ is nearly resonant with $\Delta: \Delta-\omega=-2 \varepsilon$ and $|\varepsilon| \ll \Delta$. The tunneling can only be restored at the intersection between the laser beams, where a time-dependent potential [see Eq. (2)] with frequency $\omega$ is induced. Due to the spatial variation of this potential, the resulting tunneling term is complex, whose phase factor represents the "magnetic flux" [see the sites inside the dashed rectangle, which encloses an effective magnetic flux $\phi$ ]. Here, $K(J)$ is the tunneling strength along the $x(y)$ axis, and $\varepsilon_{\mathrm{L}}=\varepsilon\left(\varepsilon_{\mathrm{R}}=-\varepsilon\right)$ the onsite energy of the atoms on the $\mathrm{L}(\mathrm{R})$ leg.

by the two rungs (meaning interleg tunnelings present) and legs, while, elsewhere, the two channels are decoupled due to an energy offset between them. The transport properties will be studied for the incident single-atom plane wave with respect to the varying of energy offset and artificial magnetic flux. If we only consider the atom propagation along the incident channel, the ladder performs as a one-channel switcher, while if both channels are considered, a two-channel router can also be realized.

Our paper is organized as follows. In Sec. II, we mainly discuss how to create the theoretical model: a two-leg ladder with only two rungs, which, together with the legs, enclose a localized artificial magnetic flux. In Sec. III, based the theoretical model, we derive the single-atom scattering coefficients between the two atomic channels. In Sec. IV, using the scattering coefficients, we discuss the single-atom switcher effect for different parameters. In Sec. V, also using the scattering coefficients, we demonstrate that the model can also be used as a single-atom router. In Sec. VI, we make some discussions in experiment. In Sec. VII, we summarize the main conclusions.

\section{CREATION OF THE TWO-LEG TWO-RUNG LADDER PENETRATED BY LOCALIZED ARTIFICIAL MAGNETIC FLUX}

The creation the two-leg ladder model can be schematically shown in Fig. 1. Concretely, we use ${ }^{87} \mathrm{Rb}$ atoms trapped by a standing wave in the $y(z)$ direction with the wave length $\lambda_{\mathrm{s}}$ $\left(\lambda_{z}\right)$, but two standing waves in the $x$ direction, which creates a double-well potential: $V_{\mathrm{s}}(x)=V_{1 x} \sin ^{2}\left(k_{1} x+\varphi / 2\right)+$ $V_{x} \sin ^{2}\left(k_{\mathrm{s}} x\right)$ with $k_{i}=2 \pi / \lambda_{i}(i=1, \mathrm{~s})$ and $\lambda_{1}=2 \lambda_{\mathrm{s}}$ [50]. The lattice depths $V_{1 x / x}$ and phase $\varphi$ are properly chosen to create an array of isolated tilted double well potentials, where each double well realizes a single ladder. In the tight-binding limit, the ladder Hamiltonian can be described by

$$
\begin{aligned}
H_{\mathrm{ld}}= & \sum_{l} \frac{\Delta}{2}\left(b_{l, \mathrm{R}}^{\dagger} b_{l, \mathrm{R}}-b_{l, \mathrm{~L}}^{\dagger} b_{l, \mathrm{~L}}\right) \\
& -\sum_{l ; q=\mathrm{L}, \mathrm{R}} J b_{l+1, q}^{\dagger} b_{l, q}-J^{\prime} \sum_{l} b_{l, \mathrm{~L}}^{\dagger} b_{l, \mathrm{R}}+\text { H.c. },
\end{aligned}
$$

where the bosonic annihilation (creation) operators $b_{l, q}\left(b_{l, q}^{\dagger}\right)$ describes the atoms at the site $l$ of the $q$ leg, and $J^{\prime}(J)$ characterizes the bare tunneling along the $x(y)$ axis. However, the tunneling $J^{\prime}$ is inhibited by the energy offset $\Delta$, suppose that $\Delta \gg J^{\prime}$ is satisfied. Besides, the reduced Plank constant has been assumed as $\hbar=1$.

The generation of the localized artificial magnetic flux is inspired by Refs. [46-49], which mainly focus on the generation of global artificial magnetic flux. The localization of the artificial magnetic flux can be achieved via controlling the thickness of two thin Raman laser beams such that the intersection of them can only cover the very four lattice sites that will enclose the artificial magnetic flux to be synthesized [see Fig. 1(a)]. The two laser beams are traveling waves with quasimomentums $\mathbf{k}_{i}=k_{i}\left(\cos \varphi_{i} \mathbf{e}_{x}+\sin \varphi_{i} \mathbf{e}_{y}\right)$ and Rabi frequencies $\Omega_{i}(i=1,2)$ which are employed to illuminate the corresponding atoms. The notation $\mathbf{e}_{x}\left(\mathbf{e}_{y}\right)$ represents the unit vector in the $x(y)$ direction. Both beams are incident in the $x y$ plane at angles $\varphi_{1}$ and $\varphi_{2}$ with respect to the $x$ axis, and couple two internal atomic energy levels $|g\rangle$ (ground state) and $|e\rangle$ (intermediate state) through large 
detunings $\delta_{i}\left(\left|\delta_{i}\right| \gg \Omega_{i}\right)$, which results in an external potential $V_{\mathrm{e}}(\mathbf{r})=\hbar \Omega \cos \left[\left(\mathbf{k}_{1}-\mathbf{k}_{\mathbf{2}}\right) \mathbf{r}+\omega t\right]|g\rangle\langle g| \quad$ with $\Omega=\frac{\Omega_{1} \Omega_{2}}{2 \delta_{1}}$. Here, the frequency difference $\omega$ can be represented by $\omega=c\left(k_{2}-k_{1}\right)=\delta_{2}-\delta_{1}$ with $|\omega| \ll\left|\delta_{i}\right|$ such that $\delta_{1} \approx \delta_{2}$. In the tight-binding limit, the external potential corresponds to the Hamiltonian

$$
H_{\mathrm{e}}(t)=\Omega \sum_{l=0,1 ; q=\mathrm{L}, \mathrm{R}} \cos \left(\phi_{l, q}+\omega t\right) b_{l, q}^{\dagger} b_{l, q},
$$

where $\phi_{l, q}=\mp \frac{k_{x} \lambda_{\mathrm{s}}}{4}+\frac{l k_{y} \lambda_{\mathrm{s}}}{2}$ and the negative (positive) sign corresponds to $q=\mathrm{L}(q=\mathrm{R})$. The quasimomentums $k_{x}$ and $k_{y}$ can be represented as $k_{x}=k_{1} \cos \varphi_{1}-k_{2} \cos \varphi_{2}$ and $k_{y}=$ $k_{1} \sin \varphi_{1}-k_{2} \sin \varphi_{2}$.

To eliminate the time-dependent Hamiltonian $H_{\mathrm{e}}(t)$, we now perform a unitary transformation

$$
U=\exp \left[-i \frac{\Omega}{\omega} \sum_{l=0,1 ; q=\mathrm{L}, \mathrm{R}} \sin \left(\phi_{l, q}+\omega t\right) b_{l, q}^{\dagger} b_{l, q}\right],
$$

which should yield the following effective Hamiltonian, that is,

$$
\begin{aligned}
H_{\mathrm{eff}}= & \sum_{l} \frac{\Delta}{2}\left(b_{l, \mathrm{R}}^{\dagger} b_{l, \mathrm{R}}-b_{l, \mathrm{~L}}^{\dagger} b_{l, \mathrm{~L}}\right) \\
& -\sum_{\substack{l \neq-1,0,1 \\
q=\mathrm{L}, \mathrm{R}}} J b_{l+1, q}^{\dagger} b_{l, q}-\sum_{l \neq 0,1} J^{\prime} b_{l, \mathrm{~L}}^{\dagger} b_{l, \mathrm{R}}+\text { H.c. } \\
& -\sum_{\substack{l=-1,0,1 \\
q=\mathrm{L}, \mathrm{R}}} J_{l, q} b_{l+1, q}^{\dagger} b_{l, q}-\sum_{l=0,1} K_{l} b_{l, \mathrm{R}}^{\dagger} b_{l, \mathrm{~L}}+\text { H.c. }
\end{aligned}
$$

The tunneling strengths in the second line of Eq. (4) remain the same as in Eq. (1), which is because Eq. (3) merely acts on the operators with $l=0,1$ and $q=\mathrm{L}, \mathrm{R}$. The new resulting tunneling strengths $J_{l, q}$ and $K_{l}$ take the following forms, i.e.,

$$
\begin{aligned}
J_{-1, q} & =J \exp \left[i \frac{\Omega}{\omega} \sin \left(\phi_{0, q}+\omega t\right)\right], \\
J_{0, q} & =J \exp \left[i \frac{\Omega}{\omega}\left(\sin \left(\phi_{1, q}+\omega t\right)-\sin \left(\phi_{0, q}+\omega t\right)\right)\right], \\
J_{1, q} & =J \exp \left[-i \frac{\Omega}{\omega} \sin \left(\phi_{1, q}+\omega t\right)\right], \\
K_{l} & =J^{\prime} \exp \left[-i \frac{\Omega}{\omega}\left(\sin \left(\phi_{l, \mathrm{~L}}+\omega t\right)-\sin \left(\phi_{l, \mathrm{R}}+\omega t\right)\right)\right] .
\end{aligned}
$$

To simplify the above tunneling strengths in Eqs. (5)-(8) (see Appendix A for derivation details), we first assume $\omega$ is nearly resonant with $\Delta$, i.e., $\Delta-\omega=-2 \varepsilon$ and $|\varepsilon| \ll \Delta$. Then, we can discard the fast-oscillating terms in the timedomain Fourier expansions of $J_{l, q}$ and $K_{l}$. After that, we continue to assume that the parameters fulfill the perturbative limit: $\frac{\Omega}{\omega} \ll 1$. Finally, the tunneling strengths in Eqs. (5)-(8) can be reduced to

$$
\begin{gathered}
J_{l, q} \equiv J, \\
K_{0}=K \exp (-i \omega t),
\end{gathered}
$$

$$
K_{1}=K \exp (i \phi) \exp (-i \omega t)
$$

where the interleg tunneling strength $K=\frac{J^{\prime} \Omega}{\omega} \cos \left(\frac{k_{x} \lambda_{\mathrm{s}}}{4}\right)$, and the artificial magnetic flux $\phi=-\frac{1}{2} k_{y} \lambda_{\mathrm{s}}=$ $-\frac{1}{2}\left(k_{1} \sin \varphi_{1}-k_{2} \sin \varphi_{2}\right) \lambda_{\mathrm{s}}$ can be tuned via adjusting the properties of Raman lasers (e.g., the angles, intensities, or quasimomentums).

Having obtained the approximate tunneling strengths in Eqs. (9)-(11), we continue to apply the unitary transformation

$$
U^{\prime}=\exp \left[-\frac{1}{2} i \omega t \sum_{l}\left(b_{l, \mathrm{R}}^{\dagger} b_{l, \mathrm{R}}-b_{l, \mathrm{~L}}^{\dagger} b_{l, \mathrm{~L}}\right)\right]
$$

to Eq. (4), such that the time dependence therein can be removed. After this transformation, we finally obtain the Hamiltonian of the two-rung two-leg ladder penetrated by a localized artificial magnetic flux [see Fig. 1(b)], i.e.,

$$
\begin{aligned}
H= & \sum_{l} \varepsilon_{\mathrm{L}} b_{l, \mathrm{~L}}^{\dagger} b_{l, \mathrm{~L}}+\varepsilon_{\mathrm{R}} b_{l, \mathrm{R}}^{\dagger} b_{l, \mathrm{R}} \\
& -\sum_{l} J\left(b_{l+1, \mathrm{~L}}^{\dagger} b_{l, \mathrm{~L}}+b_{l+1, \mathrm{R}}^{\dagger} b_{l, \mathrm{R}}\right)+\text { H.c. } \\
& -\sum_{l=0,1} K b_{0, \mathrm{~L}}^{\dagger} b_{0, \mathrm{R}}+K \exp (i \phi) b_{1, \mathrm{R}}^{\dagger} b_{1, \mathrm{~L}}+\text { H.c.. }
\end{aligned}
$$

We can see that the $\mathrm{L}$ and $\mathrm{R}$ legs, with onsite energies $\varepsilon_{\mathrm{L}}=\varepsilon$ and $\varepsilon_{\mathrm{R}}=-\varepsilon$ that produce another small energy offset, are decoupled except the sites on the loop that encloses the artificial magnetic flux $\phi$. The intraleg tunneling strength is $J$ for both legs, while the complex interleg tunneling strengths are $K$ and $K \exp (i \phi)$ at $l=0$ and $l=1$.

\section{SINGLE-ATOM TRANSPORT}

\section{A. Two-channel interaction}

In the above, we have created the two-leg bosonic ladder with only two rungs, which, together with the legs enclose an artificial magnetic flux $\phi$. Now we continue to investigate the issue of single-atom transport within this model (see Fig. 2). In particular, we concentrate on the case that the single-atom plane wave incomes from the negative sites along the $\mathrm{L}$ channel. To treat this problem, the single-atom eigenstate of the full Hamiltonian can be given by $|E\rangle=\sum_{l} u_{l, \mathrm{R}} b_{l, \mathrm{R}}^{\dagger}|0\rangle+$ $u_{l, \mathrm{~L}} b_{l, \mathrm{~L}}^{\dagger}|0\rangle$, where the coefficients $u_{l, \mu}$ for each single-atom component are constrained by $H|E\rangle=E|E\rangle$, i.e.,

$$
\begin{aligned}
E u_{l, \mathrm{R}}= & \varepsilon_{\mathrm{R}} u_{l, \mathrm{R}}-J\left(u_{l-1, \mathrm{R}}+u_{l+1, \mathrm{R}}\right) \\
& -\delta_{l, 0} K u_{l, \mathrm{~L}}-\delta_{l, 1} K e^{i \phi} u_{l, \mathrm{~L}}, \\
E u_{l, \mathrm{~L}}= & \varepsilon_{\mathrm{L}} u_{l, \mathrm{~L}}-J\left(u_{l-1, \mathrm{~L}}+u_{l+1, \mathrm{~L}}\right) \\
& -\delta_{l, 0} K u_{l, \mathrm{R}}-\delta_{l, 1} K e^{-i \phi} u_{l, \mathrm{R}} .
\end{aligned}
$$

Apparently, the interaction between both $\mathrm{L}$ and $\mathrm{R}$ channels is determined by the tunneling strength $K$ and magnetic flux $\phi$ at $l=0,1$. Hence, the incoming atom along the $\mathrm{L}$ channel will inevitably stimulate some modes in the R channel. 


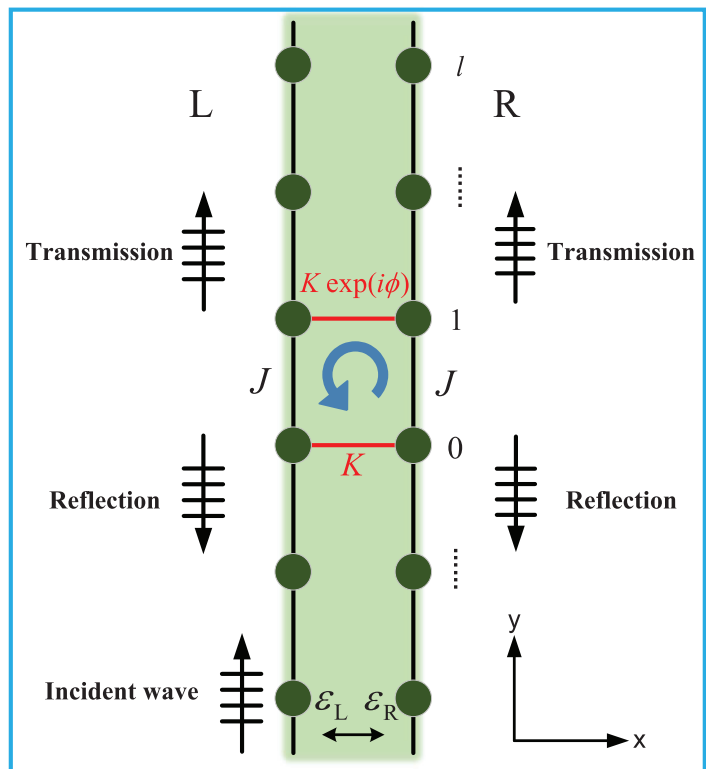

FIG. 2. Scattering process of a single-atom plane wave along the $\mathrm{L}$ leg of the ladder model running into the loop penetrated by an artificial magnetic flux $\phi$. The L and R legs are decoupled except at the sites at $l=0$ and $l=1$ that enclose the artificial magnetic flux. Away from the loop, the reflection and transmission will be stimulated in both channels. The intraleg tunneling strength is $J$ for both legs. The complex interleg tunneling strengths are respectively $K$ and $K \exp (i \phi)$ at the sites $l=0$ and $l=1$. The onsite energies for the $\mathrm{L}$ and $\mathrm{R}$ legs are respectively $\varepsilon_{\mathrm{L}}=\varepsilon$ and $\varepsilon_{\mathrm{R}}=-\varepsilon$.

\section{B. Free modes}

Now, assuming the interaction between both channels is zero, i.e., setting $K=0$ in Eqs. (14) and (15), we can obtain the free modes in both channels as

$$
u_{l, \sigma}=e^{i k_{\sigma} l},
$$

where the energy $E$ depends on $k_{\sigma}$ according to the relation

$$
E=\varepsilon_{\sigma}-2 J \cos k_{\sigma} .
$$

Here, we stress that $k_{\sigma}$ can be either real or complex depending on the regime of $E$. In detail, for either atomic channel $\sigma=\mathrm{L}$ or $\mathrm{R}$, the free modes can be classified into three cases.

(i) The energy $E$ belongs to the band $\left[\varepsilon_{\sigma}+2 J, \infty\right]$, such that $k_{\sigma}$ must be complex and can be rewritten as $k_{\sigma}=\pi+i \kappa_{\sigma}$ with $\kappa_{\sigma}$ being real. In this case, the energy turns into a hyperbolic cosine, $E=\varepsilon_{\sigma}+2 J \cosh \kappa_{\sigma}$ [see Fig. 3(a)], and the eigenstate becomes $u_{l, \sigma}=(-1)^{l} e^{-\kappa_{\sigma} l}$, a staggered decaying mode [see Fig. 3(b)].

(ii) The energy $E$ is in the band $\left(\varepsilon_{\sigma}-2 J, \varepsilon_{\sigma}+2 J\right)$, such that $k_{\sigma}$ is guaranteed to be real. In this case, the eigenstate and corresponding energy will retain their original forms as in Eqs. (16) and (17). Then,dispersive as $E=\varepsilon_{\sigma}-2 J \cos k_{\sigma}$ [see Fig. 3(c)], the eigenstate $u_{l, \sigma}=e^{i k_{\sigma} l}$ is a transmission mode [see Fig. 3(d)] with the group velocity $v_{g}=2 J \sin k_{\sigma}$.

(iii) The energy $E$ belongs to the band $\left(-\infty, \varepsilon_{\sigma}-2 J\right)$, such that $k$ is purely imaginary and can be rewritten as $k_{\sigma}=$ $i \kappa_{\sigma}$ for $\kappa_{\sigma}$ being real. In this case, the energy also turns into a hyperbolic cosine, $E=\varepsilon_{\sigma}-2 J \cosh \kappa_{\sigma}$ [see Fig. 3(e)], and
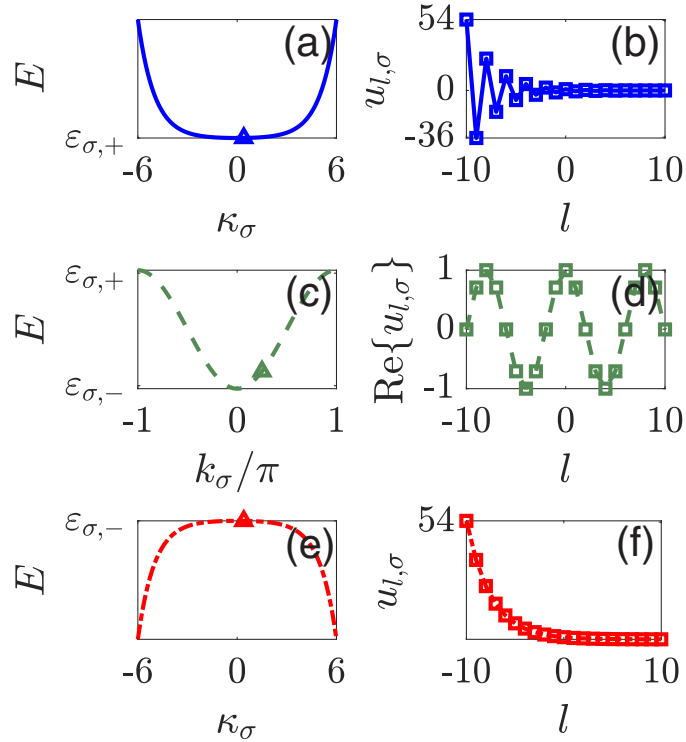

FIG. 3. Energy and eigenstates: (a) Eigenenergy $E_{\sigma}=\varepsilon_{\sigma}+$ $2 J \cosh \kappa_{\sigma}$ against $\kappa_{\sigma}(\sigma=\mathrm{L}, \mathrm{R})$. (b) Eigenstate $u_{l, \sigma}=(-1)^{l} e^{-\kappa_{\sigma} l}$ against the lattice site $l$ at $\kappa_{\sigma}=0.4$ [marked by " $\Delta$ " in (a)]. (c) Eigenenergy $E=\varepsilon_{\sigma}-2 J \cos k_{\sigma}$ against the quasimomentum $k_{\sigma}$. (d) Real part of the eigenstate $u_{l, \sigma}=e^{i k_{\sigma} l}$ against $l$ at $k_{\sigma}=$ $\frac{\pi}{4}$ [marked by " $\Delta$ " in (c)]. (e) Eigenenergy $E_{\sigma}=\varepsilon_{\sigma}-2 J \cosh \kappa_{\sigma}$ against $\kappa_{\sigma}$. (f) Eigenstate $u_{l, \sigma}=e^{-\kappa_{\sigma} l}$ against $l$ at $\kappa_{\sigma}=0.4$ marked by " $\Delta$ " in (e)]. Here, $\varepsilon_{\sigma, \pm}=\varepsilon_{\sigma} \pm 2 J$.

the eigenstate becomes $u_{l, \sigma}=e^{-\kappa_{\sigma} l}$, also a decaying mode [see Fig. 3(f)].

\section{Single-atom transport}

We consider the single-atom plane wave comes from the negative lattices along the $\mathrm{L}$ channel, which will be scattered into both $\mathrm{L}$ and $\mathrm{R}$ channels. In detail, we can express $u_{l, \mathrm{R}}$ and $u_{l, \mathrm{~L}}$ as

$$
\begin{gathered}
u_{l, \mathrm{~L}}=\left(e^{i k_{\mathrm{L}} l}+r_{\mathrm{LL}} e^{-i k_{\mathrm{L}} l}\right) \theta_{-l}+t_{\mathrm{LL}} e^{i k_{\mathrm{L}} l} \theta_{l-1}, \\
u_{l, \mathrm{R}}=r_{\mathrm{RL}} e^{-i k_{\mathrm{R}} l} \theta_{-l}+t_{\mathrm{RL}} e^{i k_{\mathrm{R}} l} \theta_{l-1},
\end{gathered}
$$

where $\theta_{l}$ is the Heaviside step function, $t_{\mathrm{LL}}\left(r_{\mathrm{LL}}\right)$ is the transmitted (reflected) amplitude, and $t_{\mathrm{RL}}\left(r_{\mathrm{RL}}\right)$ is the forward (backward) transfer amplitude.

Then, solving Eqs. (14) and (15) by assigning $l=0$, 1 , we finally obtain the scattering coefficients as

$$
\begin{aligned}
t_{\mathrm{LL}} & =D^{-1}\left(2 i \sin k_{\mathrm{L}}\right)\left[2 i \sin k_{\mathrm{R}}-\xi e^{i\left(k_{\mathrm{R}}-\phi\right)}\right], \\
t_{\mathrm{RL}} & =\frac{K}{J} D^{-1}\left(-2 i \sin k_{\mathrm{L}}\right)\left[1+e^{i\left(k_{\mathrm{L}}-k_{\mathrm{R}}+\phi\right)}\right], \\
r_{\mathrm{LL}} & =\xi D^{-1} e^{i k_{\mathrm{L}}}\left[(2 \cos \phi-\xi) e^{i k_{\mathrm{R}}}+2 \cos k_{\mathrm{L}}\right], \\
r_{\mathrm{RL}} & =\frac{K}{J} D^{-1}\left(-2 i \sin k_{\mathrm{L}}\right)\left[1+\left(e^{i \phi}-\xi\right) e^{i\left(k_{\mathrm{L}}+k_{\mathrm{R}}\right)}\right],
\end{aligned}
$$

where $\xi=\frac{K^{2}}{J^{2}}$ represents the normalized square interleg coupling strength and the denominator $D$ reads

$$
\begin{aligned}
D= & {\left[2 i \sin k_{\mathrm{L}}-\xi e^{i\left(k_{\mathrm{L}}+\phi\right)}\right]\left[2 i \sin k_{\mathrm{R}}-\xi e^{i\left(k_{\mathrm{R}}-\phi\right)}\right] } \\
& -\xi\left[1+e^{-i\left(k_{\mathrm{L}}-k_{\mathrm{R}}+\phi\right)}\right]\left[1+e^{i\left(k_{\mathrm{L}}-k_{\mathrm{R}}+\phi\right)}\right] .
\end{aligned}
$$




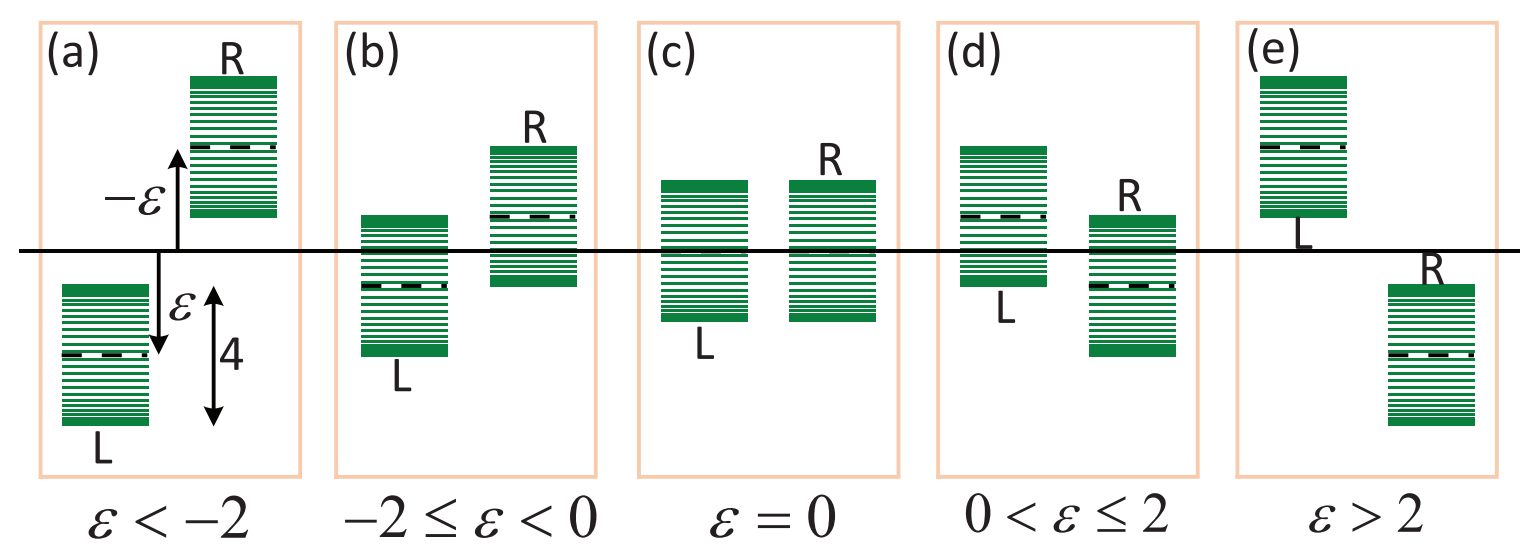

FIG. 4. Matching relations between transmission energy bands of channles $\mathrm{L}$ and $\mathrm{R}$ as the detuning $\varepsilon$ changes. Here, for simplicity, we have assumed $J=1$ such that the width of transmission energy band is $2 J=2$. If (a) $\varepsilon<-2$ or (e) $\varepsilon>2$, there is no overlap between two transmission bands. However, if (b) $-2<\varepsilon<0$ or (d) $0<\varepsilon<2$, there is partial overlap. Furthermore, if (c) $\varepsilon=0$, maximum overlap between two bands emerges.

For the lattice site $l \neq 0,1$ in Eqs. (14) and (15), the energy of the atom fulfills the condition

$$
E=\varepsilon_{\mathrm{L}}-2 J \cos k_{\mathrm{L}}=\varepsilon_{\mathrm{R}}-2 J \cos k_{\mathrm{R}}
$$

which gives the constraint of $k_{\mathrm{L}}$ and $k_{\mathrm{R}}$ in Eqs. (20)-(23). Noting that $\varepsilon_{\mathrm{L}}=\varepsilon$ and $\varepsilon_{\mathrm{R}}=-\varepsilon$ have been previously hypothesized as treating Eq. (25), we can then determine that the transmission bands of both channels can have no overlap, partial overlap, or maximum overlap if $|\varepsilon|>2 J, 0<|\varepsilon|<$ $2 J$, or $\varepsilon=0$, respectively. Figure 4 has shown the relative position of the transmission energy bands of both channels in the special case $J=1$ for different $\varepsilon$. In the case of no overlap [see Figs. 4(a) and 4(e)], the incoming atom, which must be in the transmission band, will stimulate a localized profile $\left[k_{\mathrm{R}}=i \kappa_{\mathrm{R}}\right.$ or $k_{\mathrm{R}}=i \kappa_{\mathrm{R}}+\pi$ with $\kappa_{\mathrm{R}}>0$ ], meaning no scattered atom in the $\mathrm{R}$ channel. In the case of maximum overlap [see Fig. 4(c)], the atom incoming from the L channel can be redirected into the $\mathrm{R}$ channel $\left(k_{\mathrm{R}}\right.$ is real). However, in the case of partial overlap [see Figs. 4(b) and 4(d)], the atom can either be redirected into the $\mathrm{R}$ channel or stimulate a localized profile there, just depending on the detailed energy $E$ for the incoming atom.

\section{SWITCHING THE SINGLE ATOM}

Now we only concentrate the atom propagation along the $\mathrm{L}$ channel and regard the $\mathrm{R}$ channel as an auxiliary channel, in which case, we explore the possibility to realize the atom switcher, a device which can tune the blockade and transparency of the incident atom. The blockade and transparency require that no scattered atom occur in the $\mathrm{R}$ channel, where a localized mode should be stimulated instead $\left[k_{\mathrm{R}}=i \kappa_{\mathrm{R}}\right.$ or $\left.k_{\mathrm{R}}=i \kappa_{\mathrm{R}}+\pi\right]$.

In detail, by investigating Eqs. (20) and (22) as well as noting the relation in Eq. (25), we can obtain the blockade $\left(t_{\mathrm{LL}}=0,\left|r_{\mathrm{LL}}\right|=1\right)$ condition that constrains $\phi, \varepsilon$, and $\xi$ for the incident atom at the definite quasimomentum $k_{\mathrm{L}}$, i.e.,

$$
\begin{gathered}
\phi=\pi, \\
\varepsilon=J \cos k_{\mathrm{L}} \mp \frac{J}{2}\left(\sqrt{\xi+1}+\frac{1}{\sqrt{\xi+1}}\right) .
\end{gathered}
$$

Similarly, the condition for transparency $\left(\left|t_{L L}\right|=1,\left|r_{L L}\right|=0\right)$ can also be obtained as

$$
J \cos k_{\mathrm{L}}-\frac{J}{2}\left(\frac{2 \cos k_{\mathrm{L}}}{\gamma}+\frac{\gamma}{2 \cos k_{\mathrm{L}}}\right)-\varepsilon=0,
$$

where $\gamma=\xi-2 \cos \phi \geqslant-2$ represents the resultant effect from both $\phi$ and $\xi$, and $\left|\frac{2 \cos k_{\mathrm{L}}}{\gamma}\right|<1$ must be satisfied to guarantee that $k_{\mathrm{R}}$ corresponds to a localized mode near the effective magnetic flux.

For simplicity, we set the intraleg tunneling $J=1$, which results in $\xi=K^{2}$, and the relation in Eqs. (27) and (28) can thus be illustrated in Fig. 5. We find in Fig. 5(a) that, to blockade the incoming atom at a particular quasimomentum $k_{\mathrm{L}}$, one can use a smaller $\varepsilon$ in combination with a bigger $\xi$, or vice versa. For given $\xi$, the blockade point $k_{\mathrm{L}}$ monotonically decreases as $\varepsilon$ increases. In Fig. 5(b), we also see that, if $\gamma$ is given (e.g., $\gamma=-1.6$, etc.), two transparency points may occur if $\varepsilon$ is assigned appropriately. The detailed conditions to obtain two transparency points can be obtained as $-2<\gamma<0$ and $\sqrt{1-\gamma}<|\varepsilon| \leqslant 1-\frac{\gamma}{2}$. Both Figs. 5(a) and 5 (b) are centrally symmetric about the point $\left(\varepsilon=0, k_{\mathrm{L}}=\frac{\pi}{2}\right)$. In addition, it can be verified that $\varepsilon \neq 0$ must hold, indicating the matching relations of the transmission energy bands correspond to all the subfigures in Fig. 4 except Fig. 4(c). From Fig. 5, we can conclude that the transparency and blockade points can in principle be tuned through modifying $\phi, \varepsilon$, or $\xi$.

To further gain an intuitive picture, in Fig. 6, we have plotted the transmittance $T_{\mathrm{LL}}=\left|t_{\mathrm{LL}}\right|^{2}$, reflectance $R_{\mathrm{LL}}=\left|r_{\mathrm{LL}}\right|^{2}$, and forward (backward) transfer rate $T_{\mathrm{RL}}=\left|t_{\mathrm{RL}}\right|^{2}\left(R_{\mathrm{RL}}=\right.$ $\left.\left|r_{\mathrm{RL}}\right|^{2}\right)$ as the function of $k_{\mathrm{L}}$ for the detailed parameters $\phi=$ $\pi, \xi=K^{2}=4$, but different $\varepsilon$. In such a parameter setup, the matching relations of the transmission energy bands for both channels are respectively no overlap $[\varepsilon=-2.02$, see Fig. 4(a)], partial overlap [ $\varepsilon=-0.7$, see Fig. 4(b)], maximum overlap $[\varepsilon=0$, see Fig. $4(\mathrm{c})]$, partial overlap $[\varepsilon=0.7$, see Fig. 4(d)], and no overlap [ $\varepsilon=2.02$, see Fig. 4(e)] for each column in Fig. 6 from left to right. From the curves of $T_{\mathrm{LL}}$ and $R_{\mathrm{LL}}$ in Figs. 6(a), 6(c), 6(g), and 6(i), both transparency and blockade transitions can be observed as $k_{\mathrm{L}}$ gradually 


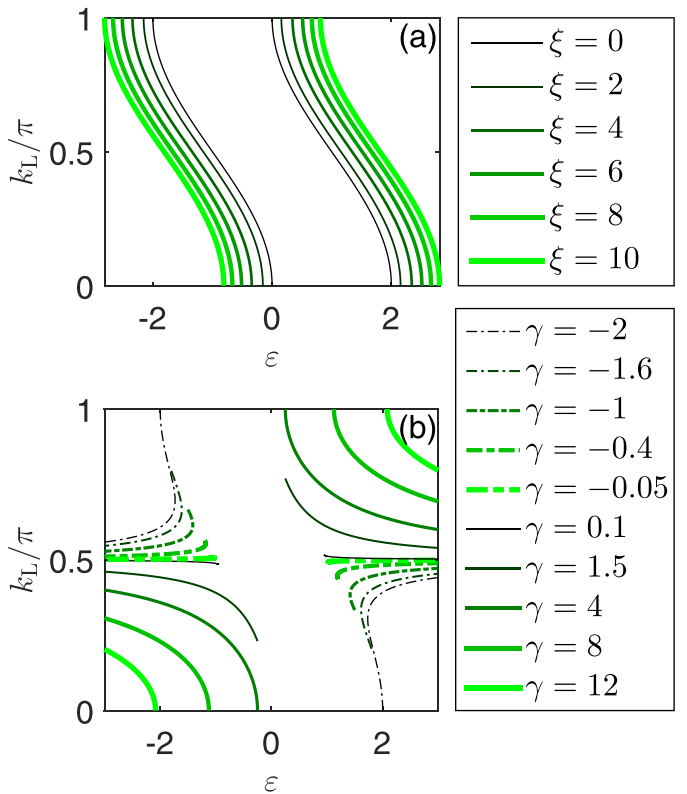

FIG. 5. Quasimomentum $k_{\mathrm{L}}$ at (a) blockade [or (b) transparency] point depending on the onsite energy $\varepsilon$ for different $\xi=\frac{K^{2}}{J^{2}}$ (or $\gamma=$ $\xi-2 \cos \phi$ ) in the special case with $J=1$. The values of $\xi$ and $\gamma$ are distinguished by both the color, thickness, and line style. Here, $J$ is the intraleg tunneling strength for both legs, while $K$ the interleg tunneling strength. Besides, $\phi$ is the artificial magnetic flux, which has taken $\pi$ in (a).

increases. When $\varepsilon$ is changed from -2.02 [see Fig. 6(a)] to -0.7 [see Fig. 6(b)], one can see that the transparency and blockade points shift from right to left. The same phenomenon also occurs when $\varepsilon$ is changed from 0.7 [see Fig. 6(g)] to 2.02 [see Fig. 6(i)]. This can be explained via Fig. 5, where we have $\xi=4$ and $\gamma=6$ for the parameters specified here. Then, we can see that both the transparency and blockade points become smaller when $\varepsilon$ increases, no matter $\varepsilon<0$ or $\varepsilon>0$. When $\varepsilon=0$ [see Fig. 6(e)], we see no transparency or blockade point, which is also consistent with the previous discussion and can be verified in Fig. 5.

In Figs. 6(b), 6(d), 6(h), and 6(j), we also note that the transfer rate $T_{\mathrm{RL}}$ can exceed 1 . On one hand, this is because $T_{\mathrm{RL}}$ and $T_{\mathrm{RL}}$ reveal the amplitudes of the localized mode stimulated in $\mathrm{R}$ channel when $k_{\mathrm{R}}$ is complex. On the other, if $k_{\mathrm{R}}$ is real, when transmission modes are stimulated in $\mathrm{R}$ channel, the atom flow conservation are subject to the relation $\tilde{T}_{\mathrm{LL}}+\tilde{R}_{\mathrm{LL}}+\tilde{T}_{\mathrm{RL}}+\tilde{R}_{\mathrm{RL}}=1$. Here, $\tilde{T}_{\mathrm{LL}}=T_{\mathrm{LL}}\left(\tilde{T}_{\mathrm{RL}}=\right.$ $\left.T_{\mathrm{RL}} \sin k_{\mathrm{R}} / \sin k_{\mathrm{L}}\right)$ and $\tilde{R}_{\mathrm{LL}}=R_{\mathrm{LL}}\left(\tilde{R}_{\mathrm{RL}}=R_{\mathrm{RL}} \sin k_{\mathrm{R}} / \sin k_{\mathrm{L}}\right)$ are respectively called the forward and backward atom flows scattered into the $\mathrm{L}(\mathrm{R})$ channel. In this case, one can conclude that $T_{\mathrm{RL}}$ can be greater than 1 once $k_{\mathrm{R}}$ is small enough, which features a sufficiently small group velocity $\left(=J \sin k_{\mathrm{R}}\right)$ in R channel. Similarly, $R_{\mathrm{RL}}$ can in principle exceed 1 as well. When $\varepsilon=0$, we have $k_{\mathrm{R}}=k_{\mathrm{L}}$, and only transmission modes are stimulated in $\mathrm{R}$ channel. Thus, we have $T_{\mathrm{LL}}+R_{\mathrm{LL}}+$ $T_{\mathrm{RL}}+R_{\mathrm{RL}}=1$, in which case, $T_{\mathrm{LL}, \mathrm{RL}}<1$ and $R_{\mathrm{LL}, \mathrm{RL}}<1$ always hold [see Figs. 6(e) and 6(f)]. In particular, one finds that $T_{\mathrm{RL}}=0$, which can be verified by Eq. (21) under the condition $k_{\mathrm{R}}=k_{\mathrm{L}}$ and $\phi=\pi$.
As shown in Fig. 7, we now investigate the transmittance $T_{\mathrm{LL}}$ and reflectance $R_{\mathrm{LL}}$ via independently modifying the values of $\varepsilon, \xi$, and $\phi$. We assign the parameters as $J=1$ and $k_{\mathrm{L}}=\frac{\pi}{4}$. Besides, in Figs. 7(a) and 7(b), $\phi=\pi$ and $K=2$; in Figs. 7(c) and 7(d), $\phi=\pi$ and $\varepsilon=-0.7$; in Figs. 7(e) and 7(f), $K=2$ and $\varepsilon$ is specified according to the blockade condition in Eq. (27), i.e., $\varepsilon=-0.6345$. We can see two (one), one (one), and one (two) blockade (transparency) points in Figs. 7(a)-7(c), respectively as $\varepsilon, \xi$, and $\phi$ varies. Note that $\phi=\pi$ is equivalent to $\phi=-\pi$ in Fig. 7(c). The number of blockade or transparency points for $\varepsilon, \xi$, or $\phi$ can be exactly predicted using Eqs. (26)-(28) (or intuitively from Fig. 5) with other parameters determined. We find that the transfer rates $T_{\mathrm{RL}}$ and $R_{\mathrm{RL}}$ [see Figs. 7(b), 7(d), and 7(f)] can also exceed 1, the reason for which is similar to that in Fig. 6. Thus, to tune the transport of the atom at a given quasimomentum, we can optionally select $\varepsilon, \xi$, or $\phi$ as the controllable parameter.

\section{ROUTING THE SINGLE ATOM}

Now we investigate the phenomenon of routing the atom from the $\mathrm{L}$ channel into the $\mathrm{R}$ one, in which case, the quasimomentums $k_{\mathrm{L}}$ and $k_{\mathrm{R}}$ are both real and thus $|\varepsilon| \leqslant 2$ must hold [see Figs. 4(b)-4(d)]. Via examining Eqs. (20) and (23), we find that when the normalized square interleg coupling strength $\xi$ and onsite energy $\varepsilon$ satisfy the conditions

$$
\begin{aligned}
& \xi=2 \cos \phi, \\
& \varepsilon=J \sin \phi,
\end{aligned}
$$

the perfect routing defined by $\tilde{T}_{\mathrm{LL}}=\tilde{R}_{\mathrm{LL}}=0$ will occur at

$$
\begin{gathered}
k_{\mathrm{R}}=\phi+\frac{\pi}{2}, \\
k_{\mathrm{L}}=\frac{\pi}{2} .
\end{gathered}
$$

Here, to guarantee that both $\xi$ and the group velocity corresponding to $k_{\mathrm{R}}$ (i.e., $J \sin k_{\mathrm{R}}$ ) are positive, there should be the constraint $-\frac{\pi}{2}<\phi<\frac{\pi}{2}$. Under the conditions in Eqs. (29)(32), the transfer amplitudes are $t_{\mathrm{RL}}=r_{\mathrm{RL}}=\frac{K}{J} \frac{i}{2 \cos \phi}$, and the corresponding atom flows are $\tilde{T}_{\mathrm{RL}}=\tilde{R}_{\mathrm{RL}}=\frac{1}{2}$.

Hereafter, we still set the intraleg tunneling strength $J=1$ for convenience. Figures 8(a)-8(c) have shown respectively how $\xi, \varepsilon$, and $k_{\mathrm{R}}$ at perfect routing [see Eqs. (29)-(31)] will change as $\phi$ varies from $-\frac{\pi}{2}$ to $\frac{\pi}{2}$. The perfect routing requires that both $\xi$ and $\varepsilon$ be sine functions of the artificial magnetic flux $\phi$. Besides, the quasimomentum of the atom outgoing from the R channel can be linearly modulated by $\phi$, despite the fact that the atom must income at the fixed quasimomentum $k_{\mathrm{L}}=\frac{\pi}{2}$ through the L channel.

If $\xi$ and $\varepsilon$ are specified according to Eqs. (29) and (30), how the atom flows vary with the quasimomentum $k_{\mathrm{L}}$ for different magnetic flux $\phi$ can be shown in Fig. 9, where, to guarantee the reality of both $k_{\mathrm{L}}$ and $k_{\mathrm{R}}$, we have constrained $\arccos \left[\frac{1}{2}(-|\varepsilon|+2+\varepsilon)\right] \leqslant k_{L} \leqslant \arccos \left[\frac{1}{2}(|\varepsilon|-2+\varepsilon)\right] . \quad$ We can observe that despite the varying of $\phi$, the perfect routing always occurs at $k_{\mathrm{L}}=\frac{\pi}{2}$. However, the quasimomentum $k_{\mathrm{R}}$ for the outgoing atom in the $\mathrm{R}$ channel will accordingly 

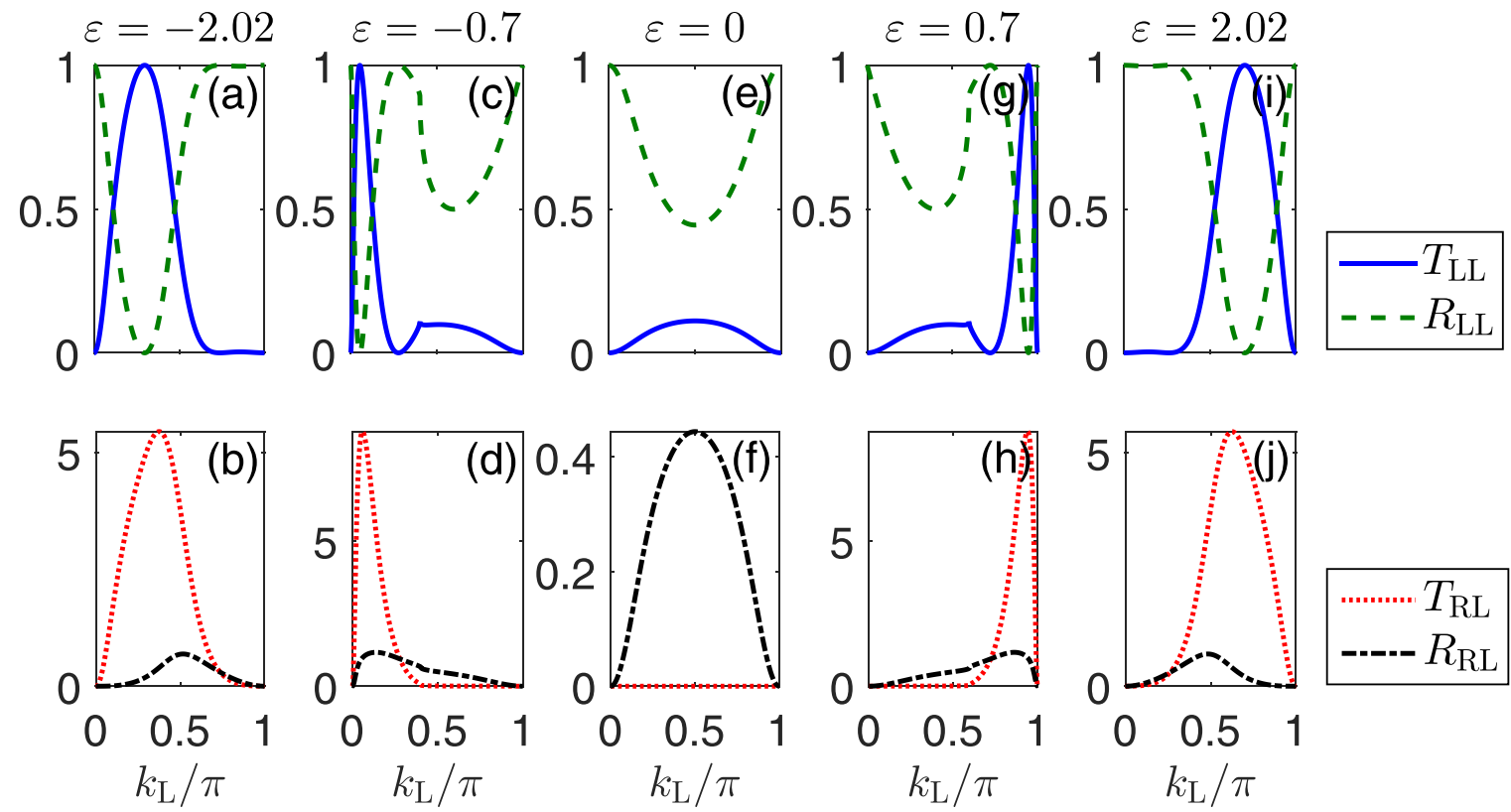

FIG. 6. Transmittance $T_{\mathrm{LL}}$, reflectance $R_{\mathrm{LL}}$, forward transfer rate $T_{\mathrm{RL}}$, and backward transfer rate $R_{\mathrm{RL}}$ against the quasimomentum $k_{\mathrm{L}}$. Here, the artificial magnetic flux $\phi=\pi$, the intraleg tunneling strength $J=1$, and the interleg tunneling strength $K=2$. The onsite energy: (a) and (b) $\varepsilon=-2.02$; (c) and (d) $\varepsilon=-0.7$; (e) and (f) $\varepsilon=0$; (g) and (h) $\varepsilon=0.7$; and (i) and (j) $\varepsilon=2.02$. In such parameter setup, the matching relations of the transmission energy bands of both channels: [(a) and (b)] no overlap [see Fig. 4(a)]; [(c) and (d)] partial overlap [see Fig. 4(b)]; [(e) and (f)] maximum overlap [see Fig. 4(c)]; [(g) and (h)] partial overlap [see Fig. 4(d)]; and [(i) and (j)] no overlap [see Fig. 4(e)].

change. It is also obvious that the two boundaries of the $k_{\mathrm{L}}$ axis are changed as $\phi$ varies, which is aroused by the variation of $\varepsilon$.
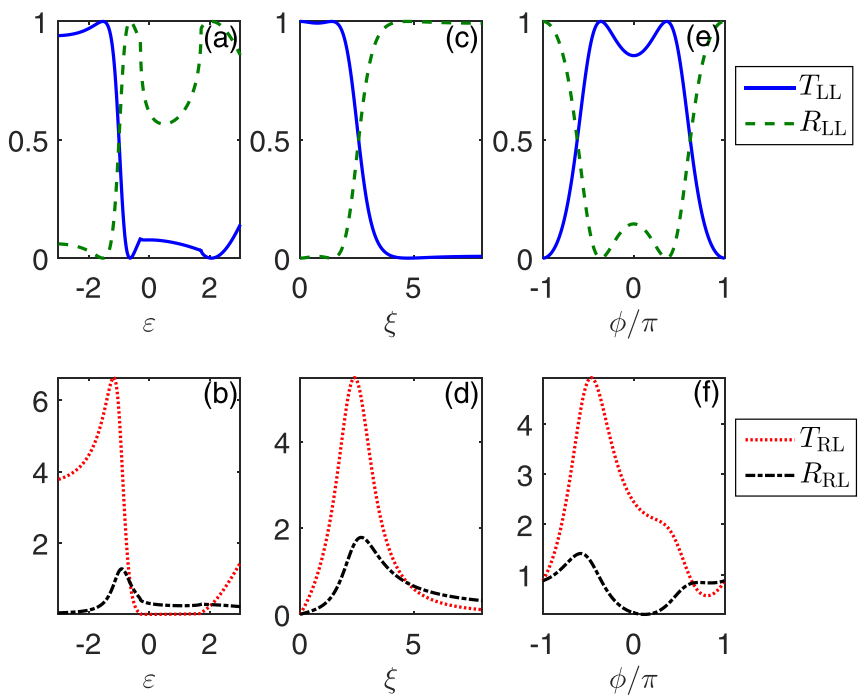

FIG. 7. Transmittance $T_{\mathrm{LL}}$ and reflectance $R_{\mathrm{LL}}$, forward transfer rate $T_{\mathrm{RL}}$, and backward transfer rate $R_{\mathrm{RL}}$ depending on the (a) onsite energy $\varepsilon$, (b) normalized square interleg coupling strength $\xi=\frac{K^{2}}{J^{2}}$, or (c) artificial magnetic flux $\phi / \pi$. Here, $K$ is the interleg coupling strength. In all plots (a)-(c), we have chosen intraleg tunneling strength $J=1$ (making $\xi=K^{2}$ ) and the quasimomentum at which the atom incomes $k_{\mathrm{L}}=\frac{\pi}{4}$. In (a) and (b), we set $\phi=\pi$, and $K=2$ (making $\xi=4$ ). In (c) and (d), $\phi=\pi$, and $\varepsilon=-0.7$. In (e) and (f), $K=2$ (making $\xi=4$ ), and $\varepsilon$ is assigned according to the blockade condition [see Eq. (27)], i.e., $\varepsilon=-0.6345$.

\section{DISCUSSIONS}

In experiment, the quasimomentum of the incident ${ }^{87} \mathrm{Rb}$ atoms can be generated via phase imprinting method [54], Bragg scattering, or simply acceleration of the matter-wave probe in an external potential. The localization magnetic flux is achieved by two laser beams whose intersection only covers four lattice sites that encloses the magnetic flux inside the ladder. The interleg tunneling strength, magnetic flux, and onsite energy can be tuned via modifying the properties of the lasers, e.g., the intensity, angles, and quasimomentums. In measurement, the motion of the atoms can be recorded by absorption imaging [55] for further analysis.

We now analyze the noise robustness of the single-atom device we have proposed here. First, to minimize the noise from the atom number fluctuation, we expect the ideal singleatom state to be generated such that the process of many-body combination that disturbs the atom number can be neglected. Second, we have hypothesized that the atoms are assumed in
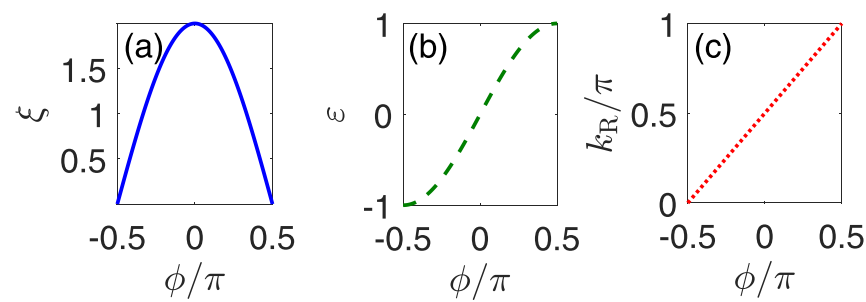

FIG. 8. (a) Normalized square interleg coupling strength $\xi$, (b) onsite energy $\varepsilon$, and (c) quasimomentum $k_{\mathrm{R}}$ in the $\mathrm{R}$ channel against the artificial magnetic flux $\phi / \pi$ in the condition of perfect routing. Here, we have assumed $J=1$ for simplicity. 


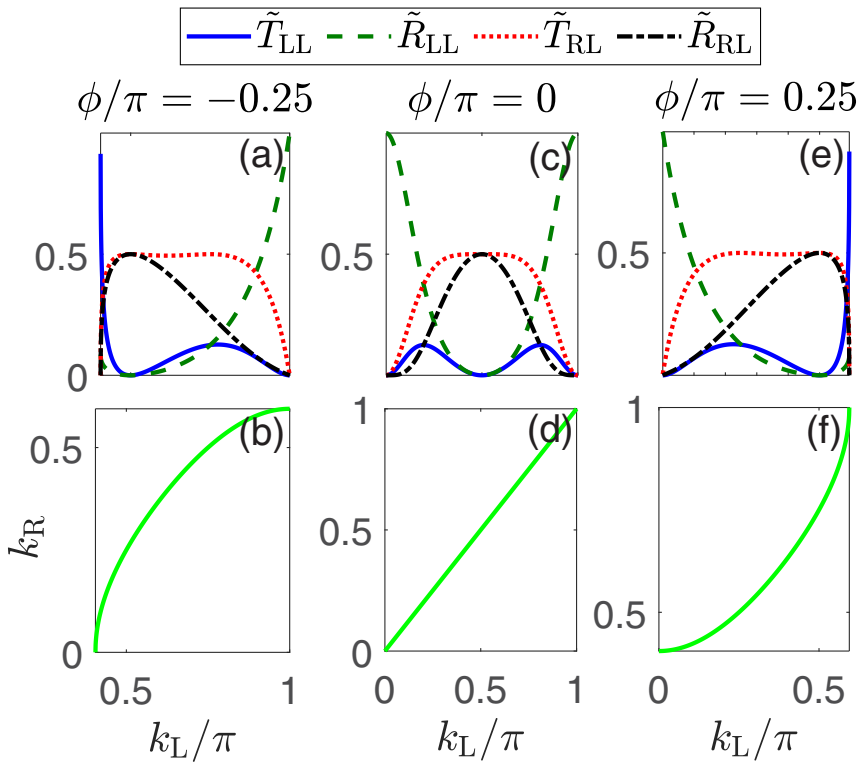

FIG. 9. Atom flows $\tilde{T}_{\mathrm{LL}}, \tilde{R}_{\mathrm{LL}}, \tilde{T}_{\mathrm{RL}}$, and $\tilde{R}_{\mathrm{RL}}$, and the corresponding quasimomentum in the $\mathrm{R}$ channel $k_{\mathrm{R}}$ against the quasimomentum of the incoming atom $k_{\mathrm{L}}$ for the artificial magnetic flux $\phi / \pi$ taking [(a) and (b)] -0.25 , [(c) and (d)] 0 , and [(e) and (f)] 0.25, respectively. In all plots (a)-(f), we have chosen the intraleg tunneling strength $J=1$ and the interleg tunneling strength $K=\sqrt{2 \cos \phi}$ such that the normalized square interleg coupling strength $\xi=$ $2 \cos \phi$, and furthermore, the onsite energy $\varepsilon=J \sin \phi$; to guarantee that both $k_{\mathrm{L}}$ and $k_{\mathrm{R}}$ are real, the range of $k_{\mathrm{L}}$ is chosen as $\arccos \left[\frac{1}{2}(-|\varepsilon|+2+\varepsilon)\right] \leqslant k_{L} \leqslant \arccos \left[\frac{1}{2}(|\varepsilon|-2+\varepsilon)\right]$.

the internal ground state. Thus, to avoid excitation to higher internal energy levels, the noise power spectrum from the control laser beam should be negligibly small at the frequency comparable to the energy level spacing. Moreover, to inhibit tunneling between legs, the laser noise should also be negligibly small at the frequency comparable to the energy offset between the two legs. For the ordinary cold atom experiment implemented at the room temperature, the thermal noise in the laser beam is too low to render significant excitation in the energy scale of atomic energy spacings or the energy offset between the ladder legs ( $\sim$ laser light frequency). Thus the intrinsic instability of the laser devices should be a limiting source of noise.

We now go deep on why many-body interactions are not involved in our present study. One reason is that we mainly focus on single-atom transport, for which, the manybody interactions are generally ineffective. Moreover, as a quantum-level phenomenon, the single-atom transport may be employed to devise more accurate atomic devices than the mean-field version with a large number of atoms. In the meanfield regime with many-body interactions, the typical method to control the incident atomic wave is to use a strong meanfield BEC (meaning a large number of atoms) [20,21]. In this method, the BEC that is used to control weak-incident-wave transport can in principle acquire accumulating momentum from incident atoms. Thus, after a series of pulses of incident waves, the strong BEC will go to an unpredictable state. This drawback should be specially studied in the future, otherwise it may prevent this mean-field method with many-body interactions from practical use.

\section{CONCLUSIONS}

In conclusion, we have proposed to generate localized artificial magnetic fields using two thin Raman laser beams intersected at a narrow region. Based on this method, we have demonstrated how to create the two-leg ladder with only two rungs, which together with the legs, enclose an artificial magnetic flux. Here, the atoms on the two legs possess opposite onsite energies that produce an energy offset. Within this model, we have explicitly investigated the single-atom transport problem. We find that the atom incoming from the left leg can experience from blockade to transparency via modifying the onsite energy, tunneling strength, or magnetic flux, which can be potentially used for a quantum switcher. Furthermore, the atom incoming from the left leg can also be perfectly routed into the right leg, when, intriguingly, the outgoing atom in the $\mathrm{R}$ channel possesses a quasimomentum that can be modulated by the magnetic flux. The result may be potentially used for the interface that controls the communication between two individual quantum devices of cold atoms.

The method can also be generalized to other artificial quantum systems, such as superconducting quantum circuit system, optical system, etc. For example, the lattice site of the ladder can be replaced with the superconducting transmon qubit circuit [56], which, in the case of weak nonlinearity and low excitation, can be modelled as photonic bosons [57]. In this implementation, the real tunneling strength can be realized via pure capacitance, while the complex tunneling strength can be achieved via the tunable coupler [58]. The noise source can be device imperfection [59] or thermal photons. The suppression of noise relies on advanced fabrication technology and ultralow working temperature. Also, the bosonic modes can be represented by optical resonator waveguides as in Ref. [60], where how to create artificial magnetic flux has also been discussed. And thus the generalization to optical system is also feasible.

Furthermore, our proposed device can also be extended to multiple channels, that is, regarding the lader as a building cell and duplicating the ladder along the rung direction. We expect the outgoing atom along each channel can be artificially tuned if we independently modify the effective magnetic flux in each individual loop. The detailed research on this topic remains an open problem.

\section{ACKNOWLEDGMENTS}

We are grateful to Ru-Quan Wang, Hai-Bin Xue, Xun-Wei $\mathrm{Xu}$, and Yu-xi Liu for helpful discussions. Y.J.Z. is supported by National Natural Science Foundation of China (NSFC) under Grants No. 11904013 and No. 11847165. W.M.L is supported by the National Key R\&D Program of China under Grant No. 2016YFA0301500, NSFC under Grant No. 61835013, SPRPCAS under Grants No. XDB01020300 and No. XDB21030300. B.L. is supported by Beijing Natural Science Foundation under Grant No. Z180007 and NSFC under Grant No. 11874002. 


\section{APPENDIX: TUNNELING STRENGTHS SIMPLIFICATION}

The tunneling strengths that need to be simplified [see Eqs. (5)-(8)] are

$$
\begin{aligned}
J_{-1, q} & =J \exp \left[i \frac{\Omega}{\omega} \sin \left(\phi_{0, q}+\omega t\right)\right], \\
J_{0, q} & =J \exp \left[i \frac{\Omega}{\omega}\left(\sin \left(\phi_{1, q}+\omega t\right)-\sin \left(\phi_{0, q}+\omega t\right)\right)\right], \\
J_{1, q} & =J \exp \left[-i \frac{\Omega}{\omega} \sin \left(\phi_{1, q}+\omega t\right)\right], \\
K_{l} & =J^{\prime} \exp \left[-i \frac{\Omega}{\omega}\left(\sin \left(\phi_{l, \mathrm{~L}}+\omega t\right)-\sin \left(\phi_{l, \mathrm{R}}+\omega t\right)\right)\right] .
\end{aligned}
$$

Noting $\phi_{l, q}=\mp \frac{k_{x} \lambda_{\mathrm{s}}}{4}+\frac{l k_{y} \lambda_{\mathrm{s}}}{2}$, with the negative (positive) sign corresponding to $q=\mathrm{L}(q=\mathrm{R})$, and using the equations of trigonometric functions, we can transform the above expressions into

$$
\begin{aligned}
J_{-1, q} & =J \exp \left[i \frac{\Omega}{\omega} \sin \left(\omega t \mp \frac{k_{x} \lambda_{\mathrm{s}}}{4}\right)\right], \\
J_{0, q} & =J \exp \left[i \frac{2 \Omega}{\omega} \cos \left(\frac{k_{y} \lambda_{\mathrm{s}}}{4}\right) \sin \left(\omega t \mp \frac{k_{x} \lambda_{\mathrm{s}}}{4}+\frac{k_{y} \lambda_{\mathrm{s}}}{4}\right)\right], \\
J_{1, q} & =J \exp \left[-i \frac{\Omega}{\omega} \sin \left(\omega t \mp \frac{k_{x} \lambda_{\mathrm{s}}}{4}+\frac{k_{y} \lambda_{\mathrm{s}}}{2}\right)\right], \\
K_{0} & =J^{\prime} \exp \left[i \frac{2 \Omega}{\omega} \cos \left(\frac{k_{x} \lambda_{\mathrm{s}}}{4}\right) \sin (\omega t)\right], \\
K_{1} & =J^{\prime} \exp \left[-i \frac{2 \Omega}{\omega} \cos \left(\frac{k_{x} \lambda_{\mathrm{s}}}{4}\right) \sin \left(\omega t+\frac{k_{y} \lambda_{\mathrm{s}}}{2}\right)\right] .
\end{aligned}
$$

To expand the above expressions into the Fourier series with respect to $t$, we can use the equation

$$
\exp (i x \sin \theta)=\sum_{N=-\infty}^{\infty} \mathcal{J}_{N}(x) \exp (i \theta),
$$

where $\mathcal{J}_{N}(x)$ represents the $N$ th Bessel function of the first kind. Then, for the tunneling strengths, we shall obtain

$$
\begin{aligned}
J_{-1, q} & =J \sum_{N=-\infty}^{\infty} \mathcal{J}_{N}\left(\frac{\Omega}{\omega}\right) \exp \left[i N\left(\omega t \mp \frac{k_{x} \lambda_{\mathrm{s}}}{4}\right)\right], \\
J_{0, q} & =J \sum_{N=-\infty}^{\infty} \mathcal{J}_{N}\left(\frac{2 \Omega}{\omega} \cos \left(\frac{k_{y} \lambda_{\mathrm{s}}}{4}\right)\right) e^{i N\left(\omega t \mp \frac{k_{x} \lambda_{\mathrm{s}}}{4}+\frac{k_{y} \lambda_{\mathrm{s}}}{4}\right)}, \\
J_{1, q} & =J \sum_{N=-\infty}^{\infty} \mathcal{J}_{N}\left(-\frac{\Omega}{\omega}\right) \exp \left[i N\left(\omega t \mp \frac{k_{x} \lambda_{\mathrm{s}}}{4}+\frac{k_{y} \lambda_{\mathrm{s}}}{2}\right)\right],
\end{aligned}
$$

$$
\begin{aligned}
& K_{0}=J^{\prime} \sum_{N=-\infty}^{\infty} \mathcal{J}_{N}\left(-\frac{2 \Omega}{\omega} \cos \left(\frac{k_{x} \lambda_{\mathrm{s}}}{4}\right)\right) \exp (i N \omega t), \\
& K_{1}=J^{\prime} \sum_{N=-\infty}^{\infty} \mathcal{J}_{N}\left(-\frac{2 \Omega}{\omega} \cos \left(\frac{k_{x} \lambda_{\mathrm{s}}}{4}\right)\right) e^{i N\left(\omega t+\frac{k_{y} \lambda_{\mathrm{s}}}{2}\right)} .
\end{aligned}
$$

To further simplify the tunneling strengths, we return to the following effective Hamiltonian [see Eq. (4)]

$$
\begin{aligned}
H_{\mathrm{eff}}= & \sum_{l} \frac{\Delta}{2}\left(b_{l, \mathrm{R}}^{\dagger} b_{l, \mathrm{R}}-b_{l, \mathrm{~L}}^{\dagger} b_{l, \mathrm{~L}}\right) \\
& -\sum_{\substack{l \neq-1,0,1 \\
q=\mathrm{L}, \mathrm{R}}} J b_{l+1, q}^{\dagger} b_{l, q}-\sum_{l \neq 0,1} J^{\prime} b_{l, \mathrm{~L}}^{\dagger} b_{l, \mathrm{R}}+\text { H.c. } \\
& -\sum_{\substack{l=-1,0,1 \\
q \\
=\mathrm{L}, \mathrm{R}}} J_{l, q} b_{l+1, q}^{\dagger} b_{l, q}-\sum_{l=0,1} K_{l} b_{l, \mathrm{R}}^{\dagger} b_{l, \mathrm{~L}}+\text { H.c. }
\end{aligned}
$$

We note that on the same leg, the onsite energies are identical, and thus in the Fourier expansions of $J_{l, q}$, the fast-oscillating terms correspond to $N \neq 0$, which should be neglected. Also, we note that the energy offset between legs is $\Delta$. We assume $\Delta-\omega=-2 \varepsilon$ with $|\varepsilon| \ll \Delta$, such that in the Fourier expansions of $K_{l}$, the fast-oscillating terms to be neglected in Eq. (A5) correspond to $N \neq-1$. Therefore, the tunneling strengths $J_{l, q}$ and $K_{l}$ can be reduced to

$$
\begin{aligned}
J_{-1, q} & =J \mathcal{J}_{0}\left(\frac{\Omega}{\omega}\right), \\
J_{0, q} & =J \mathcal{J}_{0}\left(\frac{2 \Omega}{\omega} \cos \left(\frac{k_{y} \lambda_{\mathrm{s}}}{4}\right)\right), \\
J_{1, q} & =J \mathcal{J}_{0}\left(-\frac{\Omega}{\omega}\right), \\
K_{0} & =J^{\prime} \mathcal{J}_{-1}\left(-\frac{2 \Omega}{\omega} \cos \left(\frac{k_{x} \lambda_{\mathrm{s}}}{4}\right)\right) \exp (-i \omega t), \\
K_{1} & =J^{\prime} \mathcal{J}_{-1}\left(-\frac{2 \Omega}{\omega} \cos \left(\frac{k_{x} \lambda_{\mathrm{s}}}{4}\right)\right) e^{-i\left(\omega t+\frac{k_{y} \lambda \mathrm{s}}{2}\right)} .
\end{aligned}
$$

Furthermore, we consider the perturbative limit $\frac{\Omega}{\omega} \ll 1$, which can finally give the equations as

$$
\begin{aligned}
J_{l, q} & \equiv J, \\
K_{0} & =K \exp (-i \omega t), \\
K_{1} & =K \exp [-i(\omega t-\phi)],
\end{aligned}
$$

with $K=J^{\prime} \frac{\Omega}{\omega} \cos \left(\frac{k_{x} \lambda_{\mathrm{s}}}{4}\right)$ and $\phi=-\frac{k_{y} \lambda_{\mathrm{s}}}{2}$. Here, we have used the fact that $\mathcal{J}_{0}(x) \approx 1$ and $\mathcal{J}_{-1}(x) \approx-\frac{x}{2}$ in the limit $x \ll 1$. Until now, we have obtained the simplified tunneling strengths $J_{l, q}, K_{0}$, and $K_{1}$.
[1] G. K. Brennen, C. M. Caves, P. S. Jessen, and I. H. Deutsch, Quantum Logic Gates in Optical Lattices, Phys. Rev. Lett. 82, 1060 (1999).
[2] D. Jaksch, H. J. Briegel, J. I. Cirac, C. W. Gardiner, and P. Zoller, Entanglement of Atoms via Cold Controlled Collisions, Phys. Rev. Lett. 82, 1975 (1999). 
[3] J. Pachos and P. L. Knight, Quantum Computation with a One-Dimensional Optical Lattice, Phys. Rev. Lett. 91, 107902 (2003).

[4] A. Kay, J. K. Pachos, and C. S. Adams, Graph-state preparation and quantum computation with global addressing of optical lattices, Phys. Rev. A 73, 022310 (2006).

[5] W. Hansel, P. Hommelhoff, T. W. Hansch, and J. Reichel, Bose-Einstein condensation on a microelectronic chip, Nature (London) 413, 498 (2001).

[6] J. Fortagh and C. Zimmermann, Toward atom chips, Science 307, 860 (2005).

[7] Y. Colombe, T. Steinmetz, G. Dubois, F. Linke, D. Hunger, and J. Reichel, Strong atom-field coupling for Bose-Einstein condensates in an optical cavity on a chip, Nature (London) 450, 272 (2007).

[8] M. F. Riedel, P. Bohi, Y. Li, T. W. Hansch, A. Sinatra, and P. Treutlein, Atom-chip-based generation of entanglement for quantum metrology, Nature (London) 464, 1170 (2010).

[9] T. van Zoest, N. Gaaloul, Y. Singh, H. Ahlers, W. Herr, S. T. Seidel, W. Ertmer, E. Rasel, M. Eckart, E. Kajari, S. Arnold, G. Nandi, W. P. Schleich, R. Walser, A. Vogel, K. Sengstock, K. Bongs, W. Lewoczko-Adamczyk, M. Schiemangk, T. Schuldt, A. Peters, T. Konemann, H. Muntinga, C. Lammerzahl, H. Dittus, T. Steinmetz, T. W. Hansch, and J. Reichel, BoseEinstein condensation in microgravity, Science 328, 1540 (2010).

[10] R. A. Pepino, J. Cooper, D. Z. Anderson, and M. J. Holland, Atomtronic Circuits of Diodes and Transistors, Phys. Rev. Lett. 103, 140405 (2009).

[11] S. Eckel, J. G. Lee, F. Jendrzejewski, N. Murray, C. W. Clark, C. J. Lobb, W. D. Phillips, M. Edwards, and G. K. Campbell, Hysteresis in a quantized superfluid 'atomtronic' circuit, Nature (London) 506, 200 (2014)

[12] D. Aghamalyan, M. Cominotti, M. Rizzi, D. Rossini, F. Hekking, A. Minguzzi, L.-C. Kwek, and L. Amico, Coherent superposition of current flows in an atomtronic quantum interference device, New J. Phys. 17, 045023 (2015).

[13] G. Arwas and D. Cohen, Chaos and two-level dynamics of the atomtronic quantum interference device, New J. Phys. 18, 015007 (2016).

[14] L. Amico, G. Birkl, M. Boshier, and L.-C. Kwek, Focus on atomtronics-enabled quantum technologies, New J. Phys. 19, 020201 (2017).

[15] T. A. Haase, D. H. White, D. J. Brown, I. Herrera, and M. D. Hoogerland, A versatile apparatus for two-dimensional atomtronic quantum simulation, Rev. Sci. Instrum. 88, 113102 (2017).

[16] T. Haug, J. Tan, M. Theng, R. Dumke, L.-C. Kwek, and L. Amico, Readout of the atomtronic quantum interference device, Phys. Rev. A 97, 013633 (2018).

[17] M. A. McLain and L. D. Carr, Quantum phase transition modulation in an atomtronic Mott switch, Quantum Sci. Technol 3, 035012 (2018).

[18] K. W. Wilsmann, L. H. Ymai, A. P. Tonel, J. Links, and A. Foerster, Control of tunneling in an atomtronic switching device, Commun. Phys. 1, 91 (2018).

[19] W. Lai, Y.-Q. Ma, L. Zhuang, and W. M. Liu, Photovoltaic Effect of Atomtronics Induced by an Artificial Gauge Field, Phys. Rev. Lett. 122, 223202 (2019).
[20] R. A. Vicencio, J. Brand, and S. Flach, Fano Blockade by a Bose-Einstein Condensate in an Optical Lattice, Phys. Rev. Lett. 98, 184102 (2007).

[21] Y.-J. Zhao, D. Yu, L. Zhuang, X. Gao, and W.-M. Liu, Tunable spinful matter wave valve, Sci. Rep. 9, 8653 (2019).

[22] W.-M. Liu, B. Wu, and Q. Niu, Nonlinear Effects in Interference of Bose-Einstein Condensates, Phys. Rev. Lett. 84, 2294 (2000).

[23] Z. X. Liang, Z. D. Zhang, and W. M. Liu, Dynamics of a Bright Soliton in Bose-Einstein Condensates with Time-Dependent Atomic Scattering Length in an Expulsive Parabolic Potential, Phys. Rev. Lett. 94, 050402 (2005).

[24] O. Morsch and M. Oberthaler, Dynamics of Bose-Einstein condensates in optical lattices, Rev. Mod. Phys. 78, 179 (2006).

[25] A. E. Miroshnichenko, S. Flach, and Y. S. Kivshar, Fano resonances in nanoscale structures, Rev. Mod. Phys. 82, 2257 (2010).

[26] Y. V. Kartashov, B. A. Malomed, and L. Torner, Solitons in nonlinear lattices, Rev. Mod. Phys. 83, 247 (2011).

[27] C.-C. Chien, S. Peotta, and M. Di Ventra, Quantum transport in ultracold atoms, Nat. Phys. 11, 998 (2015).

[28] U. V. Poulsen and K. Mølmer, Scattering of atoms on a BoseEinstein condensate, Phys. Rev. A 67, 013610 (2003).

[29] A. Smerzi and A. Trombettoni, Nonlinear tight-binding approximation for Bose-Einstein condensates in a lattice, Phys. Rev. A 68, 023613 (2003).

[30] C. X. Zhang, B. Zhou, Y. H. Nie, J. Q. Liang, and J. Liu, Scattering effect of atoms through a Bose-Einstein condensate in an optical lattice with a single defect, Eur. Phys. J. D 49, 161 (2008).

[31] E. Aréalo, Solitary wave solutions as a signature of the instability in the discrete nonlinear schrödinger equation, Phys. Lett. A 373, 3541 (2009).

[32] H. Hennig, J. Dorignac, and D. K. Campbell, Transfer of BoseEinstein condensates through discrete breathers in an optical lattice, Phys. Rev. A 82, 053604 (2010).

[33] X.-D. Bai, Q. Ai, M. Zhang, J. Xiong, G.-J. Yang, and F.-G. Deng, Stability and phase transition of localized modes in bose-einstein condensates with both two- and three-body interactions, Ann. Phys. 360, 679 (2015).

[34] X. D. Bai, B. A. Malomed, and F. G. Deng, Unidirectional transport of wave packets through tilted discrete breathers in nonlinear lattices with asymmetric defects, Phys. Rev. E 94, 032216 (2016).

[35] R. Qi, X.-L. Yu, Z. B. Li, and W. M. Liu, Non-Abelian Josephson Effect between Two $f=2$ Spinor Bose-Einstein Condensates in Double Optical Traps, Phys. Rev. Lett. 102, 185301 (2009).

[36] E. Tiesinga, B. J. Verhaar, and H. T. C. Stoof, Threshold and resonance phenomena in ultracold ground-state collisions, Phys. Rev. A 47, 4114 (1993).

[37] S. Inouye, M. R. Andrews, J. Stenger, H. J. Miesner, D. M. Stamper-Kurn, and W. Ketterle, Observation of Feshbach resonances in a Bose-Einstein condensate, Nature (London) 392, 151 (1998).

[38] P. O. Fedichev, Y. Kagan, G. V. Shlyapnikov, and J. T. M. Walraven, Influence of Nearly Resonant Light on the Scattering Length in Low-Temperature Atomic Gases, Phys. Rev. Lett. 77, 2913 (1996).

[39] M. Theis, G. Thalhammer, K. Winkler, M. Hellwig, G. Ruff, R. Grimm, and J. Hecker Denschlag, Tuning the Scattering 
Length with an Optically Induced Feshbach Resonance, Phys. Rev. Lett. 93, 123001 (2004).

[40] J. Lu, Z. H. Wang, and L. Zhou, T-Shaped single-photon router, Opt. Express 23, 22955 (2015).

[41] L. Zhou, L.-P. Yang, Y. Li, and C. P. Sun, Quantum Routing of Single Photons with a Cyclic Three-Level System, Phys. Rev. Lett. 111, 103604 (2013).

[42] X. Li, W.-Z. Zhang, B. Xiong, and L. Zhou, Single-photon multi-ports router based on the coupled cavity optomechanical system, Sci. Rep. 6, 39343 (2016).

[43] M. J. Collins, C. Xiong, I. H. Rey, T. D. Vo, J. He, S. Shahnia, C. Reardon, T. F. Krauss, M. J. Steel, A. S. Clark, and B. J. Eggleton, Integrated spatial multiplexing of heralded singlephoton sources, Nat. Commun. 4, 2582 (2013).

[44] T. Aoki, A. S. Parkins, D. J. Alton, C. A. Regal, B. Dayan, E. Ostby, K. J. Vahala, and H. J. Kimble, Efficient Routing of Single Photons by One Atom and a Microtoroidal Cavity, Phys. Rev. Lett. 102, 083601 (2009).

[45] I. C. Hoi, C. M. Wilson, G. Johansson, T. Palomaki, B. Peropadre, and P. Delsing, Demonstration of a Single-Photon Router in the Microwave Regime, Phys. Rev. Lett. 107, 073601 (2011).

[46] M. Aidelsburger, M. Atala, S. Nascimbène, S. Trotzky, Y. A. Chen, and I. Bloch, Experimental Realization of Strong Effective Magnetic Fields in an Optical Lattice, Phys. Rev. Lett. 107, 255301 (2011).

[47] M. Aidelsburger, M. Atala, M. Lohse, J. T. Barreiro, B. Paredes, and I. Bloch, Realization of the Hofstadter Hamiltonian with Ultracold Atoms in Optical Lattices, Phys. Rev. Lett. 111, 185301 (2013).

[48] D. Jaksch and P. Zoller, Creation of effective magnetic fields in optical lattices: The Hofstadter butterfly for cold neutral atoms, New J. Phys. 5, 56 (2003).

[49] H. Miyake, G. A. Siviloglou, C. J. Kennedy, W. C. Burton, and W. Ketterle, Realizing the Harper Hamiltonian with LaserAssisted Tunneling in Optical Lattices, Phys. Rev. Lett. 111, 185302 (2013).

[50] M. Atala, M. Aidelsburger, M. Lohse, J. T. Barreiro, B. Paredes, and I. Bloch, Observation of chiral currents with ultracold atoms in Bosonic Ladders, Nat. Phys. 10, 588 (2014).

[51] M. Mancini, G. Pagano, G. Cappellini, L. Livi, M. Rider, J. Catani, C. Sias, P. Zoller, M. Inguscio, M. Dalmonte, and L. Fallani, Observation of chiral edge states with neutral fermions in synthetic Hall ribbons, Science 349, 1510 (2015).

[52] C. Kollath, A. Sheikhan, S. Wolff, and F. Brennecke, Ultracold Fermions in a Cavity-Induced Artificial Magnetic Field, Phys. Rev. Lett. 116, 060401 (2016).

[53] F. A. An, E. J. Meier, and B. Gadway, Direct observation of chiral currents and magnetic reflection in atomic flux lattices, Sci. Adv. 3, e1602685 (2017).

[54] J. Denschlag, J. E. Simsarian, D. L. Feder, C. W. Clark, L. A. Collins, J. Cubizolles, L. Deng, E. W. Hagley, K. Helmerson, W. P. Reinhardt, S. L. Rolston, B. I. Schneider, and W. D. Phillips, Generating solitons by phase engineering of a BoseEinstein condensate, Science 287, 97 (2000).

[55] Y. Lin, K. Jimenezgarcia, and I. B. Spielman, Spin-orbitcoupled Bose-Einstein condensates, Nature (London) 471, 83 (2011).

[56] J. Koch, M. Y. Terri, J. Gambetta, A. A. Houck, D. Schuster, J. Majer, A. Blais, M. H. Devoret, S. M. Girvin, and R. J. Schoelkopf, Charge-insensitive qubit design derived from the Cooper pair box, Phys. Rev. A 76, 042319 (2007).

[57] H. Alaeian, C. W. S. Chang, M. V. Moghaddam, C. M. Wilson, E. Solano, and E. Rico, Creating lattice gauge potentials in circuit QED: The bosonic Creutz ladder, Phys. Rev. A 99, 053834 (2019).

[58] P. Roushan, C. Neill, A. Megrant, Y. Chen, R. Babbush, R. Barends, B. Campbell, Z. Chen, B. Chiaro, A. Dunsworth, A. Fowler, E. Jeffrey, J. Kelly, E. Lucero, J. Mutus, P. J. J. O’Malley, M. Neeley, C. Quintana, D. Sank, A. Vainsencher, J. Wenner, T. White, E. Kapit, H. Neven, and J. Martinis, Chiral ground-state currents of interacting photons in a synthetic magnetic field, Nat. Phys. 13, 146 (2017).

[59] A. Megrant, C. Neill, R. Barends, B. Chiaro, Y. Chen, L. Feigl, J. Kelly, E. Lucero, M. Mariantoni, P. J. J. O’Malley, D. Sank, A. Vainsencher, J. Wenner, T. C. White, Y. Yin, J. Zhao, C. J. Palmstrøm, J. M. Martinis, and A. N. Cleland, Planar superconducting resonators with internal quality factors above one million, Appl. Phys. Lett. 100, 113510 (2012).

[60] X.-W. Xu, A.-X. Chen, Y. Li, and Y.-X. Liu, Singlephoton nonreciprocal transport in one-dimensional coupled-resonator waveguides, Phys. Rev. A 95, 063808 (2017). 